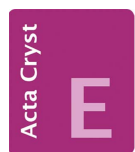

CRYSTALLOGRAPHIC COMMUNICATIONS

ISSN 2056-9890

Received 20 February 2020

Accepted 4 May 2020

Edited by L. Fabian, University of East Anglia, England

Keywords: crystal structure; solvate; pharmaceuticals; hydrogen bonding; isostructural.

CCDC reference: 2000973

Supporting information: this article has supporting information at journals.iucr.org/e

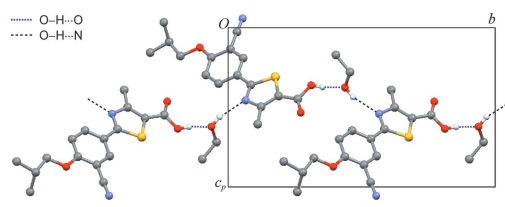

\section{Febuxostat ethanol monosolvate}

\author{
Thomas Gelbrich, ${ }^{\mathrm{a} *}$ Volker Kahlenberg, ${ }^{\mathrm{b}}$ Verena Adamer, ${ }^{\mathrm{c}}$ Sven Nerdinger ${ }^{\mathrm{c}}$ and \\ Ulrich J. Griesser ${ }^{a}$ \\ a University of Innsbruck, Institute of Pharmacy, Innrain 52, 6020 Innsbruck, Austria, ${ }^{\mathbf{b}}$ University of Innsbruck, Institute of
Mineralogy and Petrography, Innrain 52, 6020 Innsbruck, Austria, and ${ }^{\mathbf{C}}$ Sandoz GmbH, Biochemiestrasse 10, 6250
Kundl, Austria. *Correspondence e-mail: thomas.gelbrich@uibk.ac.at
}

The title compound, 2-(3-cyano-4-isobutoxyphenyl)-4-methyl-1,3-thiazole-5carboxylic acid ethanol monosolvate, $\mathrm{C}_{16} \mathrm{H}_{16} \mathrm{~N}_{2} \mathrm{O}_{3} \mathrm{~S} \cdot \mathrm{C}_{2} \mathrm{H}_{6} \mathrm{O}$, (I), displays intermolecular $\mathrm{O}-\mathrm{H} \cdots \mathrm{O}$ and $\mathrm{O}-\mathrm{H} \cdots \mathrm{N}$ bonds in which the carboxyl group of the febuxostat molecule and the hydroxyl group of the ethanol molecule serve as hydrogen-bond donor sites. These interactions result in a helical hydrogenbonded chain structure. The title structure is isostructural with a previously reported methanol analogue.

\section{Chemical context}

Febuxostat is a novel, small-molecule, non-purine-selective inhibitor of xanthine oxidase developed for the treatment of chronic gout and hyperuricemia, via oral administration (Pascual et al., 2009; Gray \& Walters-Smith, 2011; Kataoka et al., 2015). This drug is currently marketed by Takeda Pharmaceuticals Inc. under the trade name Uloric. Matsumoto et al. (1999) disclosed the existence of five solid forms of febuxostat, i.e. of the anhydrous forms $A, B$ and $C$, a methanol solvate $D$ and a hemihydrate $G$. The crystal structures of two polymorphs were reported by Maddileti et al. (2013) and Yadav et al. (2017). Additionally, solvate structures containing the febuxostat molecule and methanol (Jiang et al., 2011), acetic acid (Wu et al., 2015) or pyrdine (Zhu et al., 2009) have been described.<smiles>CCOC(C)COc1ccc(-c2nc(C)c(C(=O)O)s2)cc1C#N</smiles>

The current study was carried out as part of an investigation with the aim of establishing a modified synthetic route for febuxostat (Lutra et al., 2012), avoiding harsh conditions, toxic reagents to form the thioamide and the highly toxic cyanides. One of the key aspects of the novel route of synthesis was the introduction of a modified version of the Duff reaction (Duff \& Bills, 1932, 1934) in the first step, which finally resulted in improved overall yields compared to the original synthesis by Hasegawa (1998). 


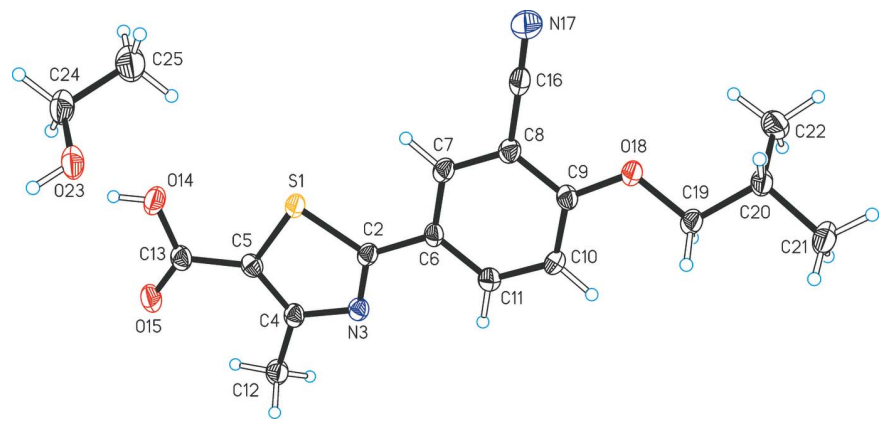

Figure 1

Asymmetric unit of (I) with displacement ellipsoids drawn at the $50 \%$ probability level and hydrogen atoms drawn as spheres of arbitrary size.

\section{Structural commentary}

The febuxostat molecule (Fig. 1) is essentially planar. This is illustrated by the fact that the mean plane defined by all its non-H atoms, except for $\mathrm{C} 22$ of the isobutyl group, results in a root-mean-square deviation for the 21 fitted atoms of only $0.0890 \AA$. Atom C22 is located at a distance of 1.498 (3) $\AA$ from this mean plane. All bond lengths and angles are in good agreement with the geometrical characteristics of previously determined febuxostat structures (see below). The relative mutual orientation of the $\mathrm{CN}$ substituent at the phenyl ring and the Me group at the thiazole ring is characterized by the torsion angle $\mathrm{S} 1-\mathrm{C} 2-\mathrm{C} 6-\mathrm{C} 7$ of $-6.5(3)^{\circ}$. This torsion is also defined as $\tau$ in the Scheme. The isobutoxy group adopts the expected extended chain geometry with $\mathrm{C} 9-\mathrm{O} 18-\mathrm{C} 19-$ $\mathrm{C} 20=175.3(2)^{\circ}$ and $\mathrm{O} 18-\mathrm{C} 19-\mathrm{C} 20-\mathrm{C} 21=170.7(2)^{\circ}$.

\section{Supramolecular features}

The carboxyl group of the febuxostat molecule is linked to the $\mathrm{OH}$ group of an EtOH molecule via an $\mathrm{O} 23-$ $\mathrm{H} 23 \cdots \mathrm{N} 3\left(-x+1, y+\frac{1}{2},-z+1\right)$ interaction. The hydroxy group of the solvent additionally serves as a hydrogen-bond donor group for an $\mathrm{O} 14-\mathrm{H} 14 \cdots \mathrm{O} 23(x-1, y, z)$ bond to a second febuxostat molecule (see Table 1). Together, these two interactions result in a hydrogen-bonded chain composed of alternating febuxostat and ethanol molecules that displays a $2_{1}$ symmetry and propagates parallel to the $b$ axis (Fig. 2). The same hydrogen-bonded structure is also present in the

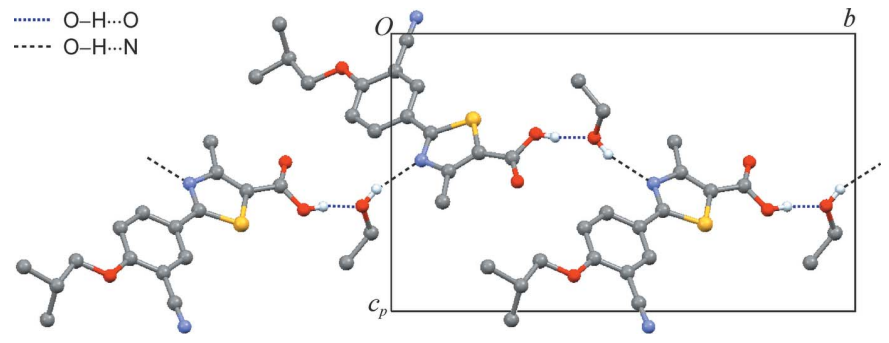

Figure 2

Hydrogen-bonded layer structure of (I), viewed along the $a$ axis.
Table 1

Hydrogen-bond geometry $\left(\AA{ }^{\circ},{ }^{\circ}\right)$.

\begin{tabular}{lllll}
\hline$D-\mathrm{H} \cdots A$ & $D-\mathrm{H}$ & $\mathrm{H} \cdots A$ & $D \cdots A$ & $D-\mathrm{H} \cdots A$ \\
\hline $\mathrm{O} 23-\mathrm{H} 23 \cdots \mathrm{N} 3^{\mathrm{i}}$ & $0.83(2)$ & $2.07(2)$ & $2.878(3)$ & $162(4)$ \\
$\mathrm{O} 14-\mathrm{H} 14 \cdots \mathrm{O} 23^{\mathrm{ii}}$ & $0.84(2)$ & $1.80(2)$ & $2.631(3)$ & $170(4)$ \\
\hline
\end{tabular}

Symmetry codes: (i) $-x+1, y+\frac{1}{2},-z+1$; (ii) $x-1, y, z$.

analogous $\mathrm{MeOH}$ solvate of febuxostat, first reported (at $296 \mathrm{~K}$ ) by Jiang et al. (2011) and redetermined by us at $173 \mathrm{~K}$ as part of this study (Gelbrich et al., 2020a). Indeed, a comparison with the program XPac (Gelbrich \& Hursthouse, 2005) reveals that the $\mathrm{EtOH}$ and $\mathrm{MeOH}$ solvates are isostructural. The comparison of corresponding geometrical parameters generated from the complete set of 22 non- $\mathrm{H}$ atomic positions in the febuxostat molecule resulted in a dissimilarity index (Gelbrich et al., 2012) of $x=3.3$, which indicates a high agreement of the febuxostat packing in the $\mathrm{EtOH}$ and $\mathrm{MeOH}$ solvates.

\section{Database survey}

Table 2 displays those entries in the Cambridge Structural Database (version 5.41, November 2019; Groom et al., 2016) that relate to crystal structures containing the febuxostat molecule. The febuxostat geometries in most of these structures are in good agreement with the parameters of (I), i.e. the torsion $\tau$ (see Scheme) typically adopts a value close to $0^{\circ}$. However, an opposite geometry with $\tau$ values close to $180^{\circ}$ has been reported for the polymorphs $\mathrm{Q}$ and $\mathrm{H} 1$, a co-crystal with 4-aminobenzoic acid and a 2-(pyridin-2-ylamino)pyridinium salt.

\section{Synthesis and crystallization}

\subsection{Synthesis}

The preparation of febuxostat was carried out according to the scheme in Fig. 3 in a modified procedure based on the original synthesis by Hasegawa (1998).

5.1.1. Ethyl 2-(3-formyl-4-hydroxyphenyl)-4-methyl-5-thiazolecarboxylate (3). Ethyl 2-(4-hydroxyphenyl)-4-methyl-5thiazole carboxylate $(\mathbf{2}, 10.0 \mathrm{~g})$ and hexamethylenetetramine $(5.86 \mathrm{~g})$ were added to trifluoroacetic acid $(100 \mathrm{ml})$. The reaction mixture was heated to reflux under stirring for $40 \mathrm{~h}$,

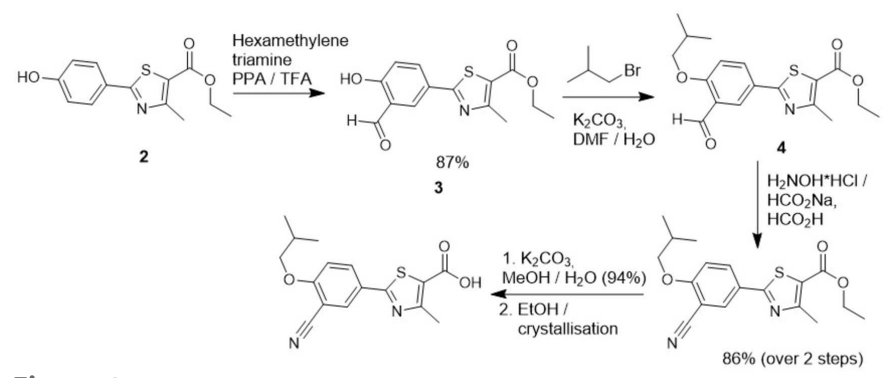

Figure 3 1 5 
Table 2

Conformation of febuxostat molecules in polymorphs and multi-component structures, indicated by the torsion angle $\tau$.

\begin{tabular}{|c|c|c|c|}
\hline Form & CSD & $\tau\left({ }^{\circ}\right)$ & Ref. \\
\hline Polymorph Q & HIQQAB & -174.1 & Maddileti et al. (2013) \\
\hline Polymorph H1 & HIQQAB02 & $\begin{array}{r}177.9 \\
-1.2\end{array}$ & Yadav et al. (2017) \\
\hline MeOH solvate ( $296 \mathrm{~K})$ & UREQOY & 5.0 & Jiang et al. (2011) \\
\hline EtOH solvate (I) & - & 4.5 & This study \\
\hline Acetic acid solvate (173 K) & CCDC 1981185 & -2.8 & Gelbrich et al. (2020b) \\
\hline Acetic acid solvate $(296 \mathrm{~K})$ & XULRUT & -3.2 & Wu et al. (2015) \\
\hline Nicotinamide co-crystal & HIQQIJ & 0.7 & Maddileti et al. (2013) \\
\hline 4-Aminobenzoic acid co-crystal & HIQQOP & -176.9 & Maddileti et al. (2013) \\
\hline Urea co-crystal & HIQQUV & 4.4 & Maddileti et al. (2013) \\
\hline Isonicotinamide co-crystal & OYADAV & -3.8 & Kang et al. (2017) \\
\hline 2-Methyl-1 $H$-imidazole salt & FAMQIW & $\begin{array}{r}-19.4 \\
13.4\end{array}$ & Zhang \& Zhang (2017) \\
\hline Imidazole salt monohydrate & KIPMAA & -5.7 & Gao et al. (2019) \\
\hline
\end{tabular}

and trifluoroacetic acid was distilled out. The obtained residue was cooled to $298 \mathrm{~K}$, water $(200 \mathrm{ml})$ was added slowly, and the slurry was stirred for $4 \mathrm{~h}$. After filtration, the product was washed and dried under vacuum to give $9.60 \mathrm{~g}$ of $\mathbf{3}$.

5.1.2. Ethyl 2-(3-formyl-4-isobutoxyphenyl)-4-methyl-5thiazolecarboxylate (4). Ethyl 2-(3-formyl-4-hydroxyphenyl)-4-methyl-5-thiazolecarboxylate $(3,350 \mathrm{~g})$, potassium carbonate $(332 \mathrm{~g})$ and isobutyl bromide $(330 \mathrm{~g})$ were added to DMF (1.75 1). The reaction mixture was heated to $383 \pm 3 \mathrm{~K}$ and stirred for $4 \mathrm{~h}$. The reaction mixture was cooled to $298 \mathrm{~K}$, and water $(0.501)$ was added slowly. The slurry was stirred for $2 \mathrm{~h}$. After filtration, the product was washed and dried under vacuum to give $389 \mathrm{~g}$ of $\left.4 .{ }^{1} \mathrm{H} \mathrm{NMR}\left(\mathrm{CDCl}_{3}\right), 400 \mathrm{MHz}\right): \delta=$ 1.079-1.101 $(d, 6 \mathrm{H}), 1.366-1.413(t, 3 \mathrm{H}), 2.185-2.230(m, 1 \mathrm{H})$, $2.769(s, 3 \mathrm{H}), 3.914-3.935(d, 2 \mathrm{H}), 4.316-4.387(q, 2 \mathrm{H}), 7.045-$ $7.074(d, 1 \mathrm{H}), 8.188-8.225(d d, 1 \mathrm{H}), 8.353-8.361(d, 1 \mathrm{H})$.

5.1.3. Ethyl 2-(3-cyano-4-isobutoxyphenyl)-4-methyl-5thiazolecarboxylate (5). Ethyl 2-(3-formyl-4-isobutoxyphenyl)-4-methyl-5-thiazolecarboxylate $(\mathbf{4}, 350 \mathrm{~g})$, sodium formate $(123 \mathrm{~g})$ and hydroxylamine hydrochloride $(84 \mathrm{~g})$ were successively added to formic acid (1.4 1). The reaction mixture was heated to reflux and stirred for $5 \mathrm{~h}$ to complete the reaction. The reaction solution was cooled to $298 \mathrm{~K}$, and water (2.8 1) was slowly added. After stirring for approximately $1 \mathrm{~h}$, the slurry was filtered, the product was washed with water and dried under vacuum to give $321 \mathrm{~g}$ of 5 . ${ }^{1} \mathrm{H} \mathrm{NMR}\left(\mathrm{CDCl}_{3}\right)$, $400 \mathrm{MHz}): \delta=1.053-1.104(d, 6 \mathrm{H}), 1.368-1.463(t, 3 \mathrm{H}), 2.164-$ $2.225(m, 1 \mathrm{H}), 2.768(s, 3 \mathrm{H}), 3.890-3.911(d, 2 \mathrm{H}), 4.324-4.395$ $(q, 2 \mathrm{H}), 6.998-7.027(d, 1 \mathrm{H}), 8$ 8.188-8.225 (dd, 1H), 8.353$8.361(d, 1 \mathrm{H})$.

5.1.4. 2-(3-Cyano-4-isobutoxyphenyl)-4-methyl-5-thiazole carboxylic acid (1). Ethyl 2-(3-cyano-4-isobutoxyphenyl)-4methyl-5-thiazolecarboxylate $(\mathbf{5}, 250 \mathrm{~g})$ and potassium carbonate $(200 \mathrm{~g})$ were successively added to a mixture of $\mathrm{MeOH}$ (7.5 l) and water $(250 \mathrm{ml})$. To complete the reaction, the solution was heated to reflux for $3 \mathrm{~h}$ under stirring. The clear solution was cooled, and vacuum was applied to distil out the solvent below $313 \mathrm{~K}$. Water (5 1) was added to the residue.
After stirring, EtOAc (2.5 l) was added. The solution was stirred, and the layers were separated. The $\mathrm{pH}$ of the aqueous solution was adjusted to $2.5 \pm 0.2$ by adding diluted hydrochloric acid solution at $313 \mathrm{~K}$. After stirring for $1 \mathrm{~h}$, the slurry was filtered, and the product was washed with water and dried under vacuum to give $215 \mathrm{~g}$ of $\mathbf{1}$.

\subsection{Crystallization}

Febuxostat $(1 \mathrm{~g})$ was dissolved in ethanol $(10 \mathrm{ml})$, which yielded a clear solution upon heating to $338 \mathrm{~K}$. After filtration, the solution was allowed to cool to room temperature, and the subsequent crystallization resulted in febuxostat ethanol solvate.

\section{Refinement}

Crystal data, data collection and structure refinement details are summarized in Table 3. All $\mathrm{H}$ atoms were identified in difference maps. Methyl $\mathrm{H}$ atoms were idealized and included as rigid groups allowed to rotate but not tip $(\mathrm{C}-\mathrm{H}=0.98 \AA)$, and their $U_{\text {iso }}$ parameters were set to $1.5 U_{\text {eq }}(\mathrm{C})$ of the parent carbon atom. $\mathrm{H}$ atoms bonded to secondary $\mathrm{CH}_{2}(\mathrm{C}-\mathrm{H}=$ $0.99 \AA)$ or tertiary $\mathrm{CH}(\mathrm{C}-\mathrm{H}=0.99 \AA)$ carbon atoms and $\mathrm{H}$ atoms bonded to $\mathrm{C}$ atoms in aromatic rings $(\mathrm{C}-\mathrm{H}=0.95 \AA)$ were positioned geometrically and refined with $U_{\text {iso }}$ set to $1.2 U_{\text {eq }}(\mathrm{C})$ of the parent carbon atom. $\mathrm{H}$ atoms in $\mathrm{OH}$ groups were identified in difference maps, refined with a distance restraint $[\mathrm{O}-\mathrm{H}=0.84(2) \AA]$ and a free $U_{\text {iso }}$ parameter. Two outliers $(\overline{474})$ and $(\overline{2}, \overline{16}, 2)$ were omitted in the final cycles of refinement.

\section{References}

Duff, J. C. \& Bills, E. J. (1932). J. Chem. Soc. pp. 1987-1988.

Duff, J. C. \& Bills, E. J. (1934). J. Chem. Soc. pp. 1305-1308.

Gao, L., Zhang, X.-R., Chen, Y.-F., Liao, Z.-L., Wang, Y.-Q. \& Zou, X.-Y. (2019). J. Mol. Struct. 1176, 633-640.

Gelbrich, T. \& Hursthouse, M. B. (2005). CrystEngComm, 7, 324-336. 
Table 3

Experimental details.

\begin{tabular}{|c|c|}
\hline \multicolumn{2}{|l|}{ Crystal data } \\
\hline Chemical formula & $\mathrm{C}_{16} \mathrm{H}_{16} \mathrm{~N}_{2} \mathrm{O}_{3} \mathrm{~S} \cdot \mathrm{C}_{2} \mathrm{H}_{6} \mathrm{O}$ \\
\hline$M_{\mathrm{r}}$ & 362.43 \\
\hline Crystal system, space group & Monoclinic, $P 2_{1}$ \\
\hline Temperature $(\mathrm{K})$ & 173 \\
\hline$a, b, c(\AA)$ & $4.7274(2), 17.7820(5), 10.7340(4)$ \\
\hline$\beta\left(^{\circ}\right)$ & $98.994(4)$ \\
\hline$V\left(\AA^{3}\right)$ & $891.23(6)$ \\
\hline$Z$ & 2 \\
\hline Radiation type & Мо $K \alpha$ \\
\hline$\mu\left(\mathrm{mm}^{-1}\right)$ & 0.21 \\
\hline Crystal size $(\mathrm{mm})$ & $0.40 \times 0.40 \times 0.36$ \\
\hline \multicolumn{2}{|l|}{ Data collection } \\
\hline Diffractometer & $\begin{array}{l}\text { Rigaku Oxford Diffraction } \\
\text { Xcalibur, Ruby, Gemini ultra }\end{array}$ \\
\hline Absorption correction & $\begin{array}{l}\text { Multi-scan (CrysAlis PRO; Rigaku } \\
\text { OD, 2015) }\end{array}$ \\
\hline$T_{\min }, T_{\max }$ & $0.760,1.000$ \\
\hline $\begin{array}{l}\text { No. of measured, independent and } \\
\text { observed }[I>2 \sigma(I)] \text { reflections }\end{array}$ & $6054,3070,2917$ \\
\hline$R_{\text {int }}$ & 0.028 \\
\hline$(\sin \theta / \lambda)_{\max }\left(\AA^{-1}\right)$ & 0.641 \\
\hline \multicolumn{2}{|l|}{ Refinement } \\
\hline$R\left[F^{2}>2 \sigma\left(F^{2}\right)\right], w R\left(F^{2}\right), S$ & $0.030,0.077,1.04$ \\
\hline No. of reflections & 3070 \\
\hline No. of parameters & 238 \\
\hline No. of restraints & 3 \\
\hline $\mathrm{H}$-atom treatment & $\begin{array}{l}\mathrm{H} \text { atoms treated by a mixture of } \\
\text { independent and constrained } \\
\text { refinement }\end{array}$ \\
\hline$\Delta \rho_{\max }, \Delta \rho_{\min }\left(\mathrm{e} \AA^{-3}\right)$ & $0.26,-0.17$ \\
\hline Absolute structure & $\begin{array}{l}\text { Flack } x \text { determined using } 1046 \\
\text { quotients }\left[\left(I^{+}\right)-\left(I^{\prime}\right)\right] /\left[\left(I^{+}\right)+\left(I^{-}\right)\right] \\
\text {(Parsons et al., 2013). }\end{array}$ \\
\hline Absolute structure parameter & $-0.02(4)$ \\
\hline
\end{tabular}

Computer programs: CrysAlis PRO (Rigaku OD, 2015), SHELXT (Sheldrick, 2015a), SHELXL2014/6 (Sheldrick, 2015b), XP in SHELXTL (Sheldrick, 2008), Mercury (Macrae et al., 2020), PLATON (Spek, 2020) and publCIF (Westrip, 2010).

Gelbrich, T., Kahlenberg, V., Adamer, V. \& Griesser, U. J. (2020a). Private Communication (CCDC reference 1981184). CCDC, Cambridge, England.
Gelbrich, T., Kahlenberg, V., Adamer, V. \& Griesser, U. J. (2020b). Private Communication (CCDC reference 1981185). CCDC, Cambridge, England.

Gelbrich, T., Threlfall, T. L. \& Hursthouse, M. B. (2012). CrystEngComm, 14, 5454-5464.

Gray, C. L. \& Walters-Smith, N. E. (2011). Am. J. Health Syst. Pharm. 68, 389-398.

Groom, C. R., Bruno, I. J., Lightfoot, M. P. \& Ward, S. C. (2016). Acta Cryst. B72, 171-179.

Hasegawa, M. (1998). Heterocycles, 47, 857-864.

Jiang, Q.-Y., Qian, J.-J., Gu, J.-M., Tang, G.-P. \& Hu, X.-R. (2011). Acta Cryst. E67, o1232.

Kang, Y., Gu, J. \& Hu, X. (2017). J. Mol. Struct. 1130, 480-486.

Kataoka, H., Yang, K. \& Rock, K. L. (2015). Eur. J. Pharmacol. 746, 174-179.

Lutra, P., Khan, R., Salunkhe, D. \& Nasir, A. (2012). Int. Patent Appl. WO 2012131590 (A1).

Macrae, C. F., Sovago, I., Cottrell, S. J., Galek, P. T. A., McCabe, P., Pidcock, E., Platings, M., Shields, G. P., Stevens, J. S., Towler, M. \& Wood, P. A. (2020). J. Appl. Cryst. 53, 226-235.

Maddileti, D., Jayabun, S. K. \& Nangia, A. (2013). Cryst. Growth Des. 13, 3188-3196.

Matsumoto, K., Watanabe, K., Hiramatsu, T. \& Kitamura, M. (1999). Int. Patent Appl. WO 1999065885 (A1).

Parsons, S., Flack, H. D. \& Wagner, T. (2013). Acta Cryst. B69, 249 259.

Pascual, E., Sivera, F., Yasothan, U. \& Kirkpatrick, P. (2009). Nat. Rev. Drug Discov. 8, 191-192.

Rigaku OD (2015). CrysAlis PRO. Rigaku Corporation, Tokyo, Japan.

Sheldrick, G. M. (2008). Acta Cryst. A64, 112-122.

Sheldrick, G. M. (2015a). Acta Cryst. A71, 3-8.

Sheldrick, G. M. (2015b). Acta Cryst. C71, 3-8.

Spek, A. L. (2020). Acta Cryst. E76, 1-11.

Westrip, S. P. (2010). J. Appl. Cryst. 43, 920-925.

Wu, M., Hu, X.-R., Gu, J.-M. \& Tang, G.-P. (2015). Acta Cryst. E71, o295-o296.

Yadav, J. A., Khomane, K. S., Modi, S. R., Ugale, B., Yadav, R. N., Nagaraja, C. M., Kumar, N. \& Bansal, A. K. (2017). Mol. Pharm. 14, 866-874.

Zhang, X.-R. \& Zhang, L. (2017). J. Mol. Struct. 1137, 328-334.

Zhu, X., Wang, Y. \& Lu, T. (2009). Acta Cryst. E65, o2603. 


\section{supporting information}

Acta Cryst. (2020). E76, 816-819 [https://doi.org/10.1107/S2056989020006076]

\section{Febuxostat ethanol monosolvate}

\section{Thomas Gelbrich, Volker Kahlenberg, Verena Adamer, Sven Nerdinger and Ulrich J. Griesser}

\section{Computing details}

Data collection: CrysAlis PRO (Rigaku OD, 2015); cell refinement: CrysAlis PRO (Rigaku OD, 2015); data reduction: CrysAlis PRO (Rigaku OD, 2015); program(s) used to solve structure: SHELXT (Sheldrick, 2015a); program(s) used to refine structure: SHELXL2014/6 (Sheldrick, 2015b); molecular graphics: XP in SHELXTL (Sheldrick, 2008) and Mercury (Macrae et al., 2020); software used to prepare material for publication: PLATON (Spek, 2020) and publCIF (Westrip, 2010).

2-[3-Cyano-4-(2-methylpropoxy)phenyl]-4-methyl-1,3-thiazole-5-carboxylic acid ethanol monosolvate

\section{Crystal data}

$$
\begin{aligned}
& \mathrm{C}_{16} \mathrm{H}_{16} \mathrm{~N}_{2} \mathrm{O}_{3} \mathrm{~S} \cdot \mathrm{C}_{2} \mathrm{H}_{6} \mathrm{O} \\
& M_{r}=362.43 \\
& \text { Monoclinic, } P 2_{1} \\
& a=4.7274(2) \AA \\
& b=17.7820(5) \AA \\
& c=10.7340(4) \AA \\
& \beta=98.994(4)^{\circ} \\
& V=891.23(6) \AA^{3} \\
& Z=2
\end{aligned}
$$

\section{Data collection}

Rigaku Oxford Diffraction Xcalibur, Ruby, Gemini ultra diffractometer

Radiation source: fine-focus sealed X-ray tube, Enhance (Mo) X-ray Source

Graphite monochromator

Detector resolution: 10.3575 pixels $\mathrm{mm}^{-1}$

$\omega$ scans

Absorption correction: multi-scan

CrysAlisPro (Rigaku OD, 2015)

\section{Refinement}

Refinement on $F^{2}$

Least-squares matrix: full

$R\left[F^{2}>2 \sigma\left(F^{2}\right)\right]=0.030$

$w R\left(F^{2}\right)=0.077$

$S=1.04$

3070 reflections

238 parameters

3 restraints
$F(000)=384$

$D_{\mathrm{x}}=1.351 \mathrm{Mg} \mathrm{m}^{-3}$

Mo $K \alpha$ radiation, $\lambda=0.71073 \AA$

Cell parameters from 2681 reflections

$\theta=2.3-28.6^{\circ}$

$\mu=0.21 \mathrm{~mm}^{-1}$

$T=173 \mathrm{~K}$

Prism, colourless

$0.40 \times 0.40 \times 0.36 \mathrm{~mm}$

$T_{\min }=0.760, T_{\max }=1.000$

6054 measured reflections

3070 independent reflections

2917 reflections with $I>2 \sigma(I)$

$R_{\text {int }}=0.028$

$\theta_{\max }=27.1^{\circ}, \theta_{\min }=1.9^{\circ}$

$h=-5 \rightarrow 6$

$k=-17 \rightarrow 22$

$l=-12 \rightarrow 13$

Primary atom site location: structure-invariant direct methods

Secondary atom site location: difference Fourier map

Hydrogen site location: mixed

$\mathrm{H}$ atoms treated by a mixture of independent and constrained refinement

$w=1 /\left[\sigma^{2}\left(F_{\mathrm{o}}^{2}\right)+(0.0439 P)^{2}+0.0696 P\right]$ where $P=\left(F_{\mathrm{o}}^{2}+2 F_{\mathrm{c}}^{2}\right) / 3$ 
$(\Delta / \sigma)_{\max }<0.001$

$\Delta \rho_{\max }=0.26 \mathrm{e} \AA^{-3}$

$\Delta \rho_{\min }=-0.17 \mathrm{e} \AA^{-3}$
Absolute structure: Flack $x$ determined using 1046 quotients $\left[\left(I^{+}\right)-\left(I^{-}\right)\right] /\left[\left(I^{+}\right)+\left(I^{-}\right)\right]$(Parsons et al., 2013).

Absolute structure parameter: $-0.02(4)$

\section{Special details}

Geometry. All esds (except the esd in the dihedral angle between two 1.s. planes) are estimated using the full covariance matrix. The cell esds are taken into account individually in the estimation of esds in distances, angles and torsion angles; correlations between esds in cell parameters are only used when they are defined by crystal symmetry. An approximate (isotropic) treatment of cell esds is used for estimating esds involving l.s. planes.

Fractional atomic coordinates and isotropic or equivalent isotropic displacement parameters $\left(\AA^{2}\right)$

\begin{tabular}{|c|c|c|c|c|}
\hline & $x$ & $y$ & $z$ & $U_{\text {iso }} * / U_{\text {eq }}$ \\
\hline S1 & $0.32087(12)$ & $0.17683(3)$ & $0.31163(5)$ & $0.02221(15)$ \\
\hline $\mathrm{C} 2$ & $0.3894(5)$ & $0.08540(14)$ & $0.3610(2)$ & $0.0195(5)$ \\
\hline N3 & $0.2673(4)$ & $0.06576(12)$ & $0.45794(18)$ & 0.0199 (4) \\
\hline $\mathrm{C} 4$ & $0.1127(5)$ & $0.12385(14)$ & $0.4979(2)$ & $0.0210(5)$ \\
\hline $\mathrm{C} 5$ & $0.1165(5)$ & $0.18918(14)$ & $0.4308(2)$ & $0.0209(5)$ \\
\hline C6 & $0.5737(5)$ & $0.03525(14)$ & 0.3007 (2) & $0.0188(5)$ \\
\hline $\mathrm{C} 7$ & $0.6801(5)$ & $0.05673(15)$ & $0.1922(2)$ & $0.0211(5)$ \\
\hline H7 & 0.6309 & 0.1045 & 0.1555 & $0.025^{*}$ \\
\hline $\mathrm{C} 8$ & $0.8571(5)$ & $0.00904(14)$ & $0.1371(2)$ & $0.0208(5)$ \\
\hline $\mathrm{C} 9$ & $0.9280(5)$ & $-0.06253(15)$ & $0.1882(2)$ & $0.0207(5)$ \\
\hline $\mathrm{C} 10$ & $0.8252(5)$ & $-0.08344(15)$ & $0.2980(2)$ & $0.0231(5)$ \\
\hline H10 & 0.8757 & -0.1309 & 0.3357 & $0.028^{*}$ \\
\hline C11 & $0.6499(5)$ & $-0.03524(14)$ & $0.3523(2)$ & $0.0225(5)$ \\
\hline H11 & 0.5797 & -0.0505 & 0.4266 & $0.027^{*}$ \\
\hline $\mathrm{C} 12$ & $-0.0406(5)$ & $0.11107(16)$ & $0.6073(2)$ & $0.0269(6)$ \\
\hline $\mathrm{H} 12 \mathrm{~A}$ & 0.0985 & 0.1093 & 0.6852 & $0.040 *$ \\
\hline H12B & -0.1448 & 0.0633 & 0.5962 & $0.040^{*}$ \\
\hline $\mathrm{H} 12 \mathrm{C}$ & -0.1761 & 0.1522 & 0.6125 & $0.040^{*}$ \\
\hline $\mathrm{C} 13$ & $-0.0224(5)$ & $0.26111(15)$ & $0.4499(2)$ & $0.0234(5)$ \\
\hline $\mathrm{O} 14$ & $0.0142(5)$ & $0.31184(12)$ & $0.36340(19)$ & $0.0339(5)$ \\
\hline H14 & $-0.062(8)$ & $0.3537(16)$ & $0.375(4)$ & $0.072(13)^{*}$ \\
\hline O15 & $-0.1558(4)$ & $0.27251(11)$ & $0.53494(18)$ & $0.0345(5)$ \\
\hline $\mathrm{C} 16$ & $0.9755(6)$ & $0.03373(15)$ & $0.0278(2)$ & $0.0259(6)$ \\
\hline N17 & $1.0707(5)$ & $0.05481(15)$ & $-0.0569(2)$ & $0.0387(6)$ \\
\hline $\mathrm{O} 18$ & $1.0943(4)$ & $-0.10584(10)$ & $0.12590(16)$ & 0.0248 (4) \\
\hline C19 & $1.1767(5)$ & $-0.17866(15)$ & $0.1797(2)$ & $0.0227(5)$ \\
\hline H19A & 1.0042 & -0.2076 & 0.1919 & $0.027^{*}$ \\
\hline H19B & 1.2988 & -0.1724 & 0.2627 & $0.027^{*}$ \\
\hline $\mathrm{C} 20$ & $1.3396(5)$ & $-0.22023(14)$ & $0.0906(2)$ & $0.0236(5)$ \\
\hline $\mathrm{H} 20$ & 1.4987 & -0.1875 & 0.0709 & $0.028^{*}$ \\
\hline $\mathrm{C} 21$ & $1.4684(6)$ & $-0.29130(17)$ & $0.1579(3)$ & $0.0327(6)$ \\
\hline $\mathrm{H} 21 \mathrm{~A}$ & 1.5817 & -0.2775 & 0.2390 & $0.049 *$ \\
\hline H21B & 1.5919 & -0.3164 & 0.1054 & $0.049^{*}$ \\
\hline $\mathrm{H} 21 \mathrm{C}$ & 1.3139 & -0.3254 & 0.1722 & $0.049 *$ \\
\hline C22 & $1.1480(7)$ & $-0.24066(17)$ & $-0.0320(3)$ & $0.0336(6)$ \\
\hline
\end{tabular}




\begin{tabular}{lllll} 
H22A & 1.0040 & -0.2773 & -0.0148 & $0.050^{*}$ \\
H22B & 1.2643 & -0.2625 & -0.0907 & $0.050^{*}$ \\
H22C & 1.0519 & -0.1953 & -0.0694 & $0.050^{*}$ \\
O23 & $0.7299(4)$ & $0.43799(11)$ & $0.37730(18)$ & $0.0347(5)$ \\
H23 & $0.741(8)$ & $0.4681(18)$ & $0.437(3)$ & $0.053(11)^{*}$ \\
C24 & $0.4973(6)$ & $0.45692(16)$ & $0.2805(3)$ & $0.0347(7)$ \\
H24A & 0.5205 & 0.5091 & 0.2515 & $0.042^{*}$ \\
H24B & 0.3143 & 0.4538 & 0.3142 & $0.042^{*}$ \\
C25 & $0.4914(7)$ & $0.40395(19)$ & $0.1727(3)$ & $0.0440(8)$ \\
H25A & 0.4835 & 0.3521 & 0.2031 & $0.066^{*}$ \\
H25B & 0.6647 & 0.4107 & 0.1343 & $0.066^{*}$ \\
H25C & 0.3222 & 0.4141 & 0.1097 & $0.066^{*}$ \\
\hline
\end{tabular}

Atomic displacement parameters $\left(\AA^{2}\right)$

\begin{tabular}{|c|c|c|c|c|c|c|}
\hline & $U^{11}$ & $U^{22}$ & $U^{33}$ & $U^{12}$ & $U^{13}$ & $U^{23}$ \\
\hline $\mathrm{S} 1$ & 0.0281 & $0.0153(3)$ & $0.0243(3)$ & $0.0024(3)$ & 0.0077 (2) & $0.0010(3)$ \\
\hline $\mathrm{C} 2$ & $0.0205(11)$ & $0.0154(12)$ & $0.0214(11)$ & $-0.0005(9)$ & $-0.0003(10)$ & $-0.0001(10)$ \\
\hline N3 & $0.0210(10)$ & $0.0168(11)$ & $0.0219(10)$ & $-0.0017(8)$ & $0.0028(8)$ & $-0.0008(8)$ \\
\hline $\mathrm{C} 4$ & $0.0212(12)$ & 0.0173 (12) & $0.0234(12)$ & $-0.0013(10)$ & $-0.0002(10)$ & $-0.0031(10)$ \\
\hline $\mathrm{C} 5$ & $0.0207(11)$ & 0.0209 (14) & $0.0211(11)$ & $-0.0028(10)$ & $0.0034(9)$ & $-0.0034(10)$ \\
\hline C6 & $0.0199(11)$ & 0.0149 (12) & $0.0211(11)$ & $0.0003(10)$ & $0.0014(9)$ & $-0.0033(9)$ \\
\hline $\mathrm{C} 7$ & $0.0237(12)$ & $0.0146(12)$ & $0.0244(12)$ & $-0.0014(10)$ & $0.0018(10)$ & $0.0006(10)$ \\
\hline $\mathrm{C} 8$ & $0.0231(12)$ & $0.0164(12)$ & $0.0225(12)$ & $-0.0018(10)$ & $0.0025(10)$ & $-0.0008(10)$ \\
\hline C9 & $0.0233(12)$ & $0.0167(12)$ & $0.0223(12)$ & $-0.0004(10)$ & $0.0040(10)$ & $-0.0033(10)$ \\
\hline $\mathrm{C} 10$ & $0.0303(14)$ & $0.0151(13)$ & $0.0240(12)$ & $0.0027(10)$ & $0.0046(10)$ & $0.0029(10)$ \\
\hline C11 & $0.0259(13)$ & $0.0200(13)$ & $0.0219(12)$ & $0.0001(11)$ & $0.0045(10)$ & $-0.0006(10)$ \\
\hline $\mathrm{C} 12$ & 0.0309 (14) & $0.0218(14)$ & $0.0296(13)$ & $0.0002(11)$ & 0.0095 (11) & $-0.0001(11)$ \\
\hline $\mathrm{C} 13$ & $0.0255(12)$ & $0.0192(13)$ & 0.0245 (13) & $-0.0024(11)$ & $0.0006(11)$ & $-0.0046(10)$ \\
\hline $\mathrm{O} 14$ & $0.0474(12)$ & $0.0196(10)$ & $0.0387(11)$ & $0.0099(9)$ & $0.0189(9)$ & $0.0045(9)$ \\
\hline $\mathrm{O} 15$ & $0.0518(12)$ & $0.0232(10)$ & $0.0327(10)$ & $0.0046(9)$ & $0.0191(9)$ & $-0.0043(8)$ \\
\hline $\mathrm{C} 16$ & $0.0314(13)$ & $0.0166(13)$ & 0.0305 (14) & $0.0045(11)$ & $0.0072(12)$ & $-0.0009(11)$ \\
\hline N17 & $0.0512(15)$ & $0.0318(15)$ & 0.0377 (13) & $0.0033(12)$ & $0.0210(12)$ & $0.0081(12)$ \\
\hline $\mathrm{O} 18$ & $0.0328(9)$ & $0.0175(9)$ & $0.0260(9)$ & $0.0062(8)$ & $0.0105(8)$ & $0.0031(7)$ \\
\hline C19 & 0.0267 (13) & $0.0162(13)$ & $0.0259(12)$ & $0.0018(10)$ & $0.0068(10)$ & $0.0024(10)$ \\
\hline $\mathrm{C} 20$ & $0.0264(12)$ & $0.0172(13)$ & 0.0292 (13) & $0.0025(10)$ & 0.0108 (11) & $0.0018(10)$ \\
\hline $\mathrm{C} 21$ & 0.0333 (14) & 0.0225 (14) & $0.0452(16)$ & $0.0077(12)$ & $0.0146(13)$ & $0.0072(13)$ \\
\hline $\mathrm{C} 22$ & 0.0447 (16) & $0.0284(16)$ & $0.0290(14)$ & $-0.0006(13)$ & 0.0099 (12) & $-0.0043(12)$ \\
\hline $\mathrm{O} 23$ & $0.0465(12)$ & $0.0218(10)$ & $0.0345(11)$ & $0.0092(9)$ & $0.0025(9)$ & $-0.0071(9)$ \\
\hline $\mathrm{C} 24$ & $0.0395(16)$ & $0.0237(15)$ & $0.0416(16)$ & $0.0086(13)$ & 0.0078 (13) & $-0.0036(12)$ \\
\hline $\mathrm{C} 25$ & 0.0536 (19) & $0.0343(18)$ & $0.0424(17)$ & $0.0044(15)$ & $0.0020(15)$ & $-0.0067(14)$ \\
\hline
\end{tabular}

Geometric parameters $\left(\AA,{ }^{\circ}\right)$

\begin{tabular}{lllr}
\hline $\mathrm{S} 1-\mathrm{C} 2$ & $1.725(3)$ & $\mathrm{O} 14-\mathrm{H} 14$ & $0.84(2)$ \\
$\mathrm{S} 1-\mathrm{C} 5$ & $1.733(2)$ & $\mathrm{C} 16-\mathrm{N} 17$ & $1.139(3)$ \\
$\mathrm{C} 2-\mathrm{N} 3$ & $1.313(3)$ & $\mathrm{O} 18-\mathrm{C} 19$ & $1.446(3)$ \\
$\mathrm{C} 2-\mathrm{C} 6$ & $1.466(3)$ & $\mathrm{C} 19-\mathrm{C} 20$ & $1.511(3)$
\end{tabular}




\begin{tabular}{|c|c|c|c|}
\hline $\mathrm{N} 3-\mathrm{C} 4$ & $1.372(3)$ & C19-H19A & 0.9900 \\
\hline $\mathrm{C} 4-\mathrm{C} 5$ & $1.369(4)$ & C19-H19B & 0.9900 \\
\hline $\mathrm{C} 4-\mathrm{C} 12$ & $1.491(3)$ & $\mathrm{C} 20-\mathrm{C} 22$ & $1.520(4)$ \\
\hline $\mathrm{C} 5-\mathrm{C} 13$ & $1.467(4)$ & $\mathrm{C} 20-\mathrm{C} 21$ & $1.534(4)$ \\
\hline $\mathrm{C} 6-\mathrm{C} 7$ & $1.393(3)$ & $\mathrm{C} 20-\mathrm{H} 20$ & 1.0000 \\
\hline $\mathrm{C} 6-\mathrm{C} 11$ & $1.395(3)$ & $\mathrm{C} 21-\mathrm{H} 21 \mathrm{~A}$ & 0.9800 \\
\hline $\mathrm{C} 7-\mathrm{C} 8$ & $1.387(3)$ & $\mathrm{C} 21-\mathrm{H} 21 \mathrm{~B}$ & 0.9800 \\
\hline $\mathrm{C} 7-\mathrm{H} 7$ & 0.9500 & $\mathrm{C} 21-\mathrm{H} 21 \mathrm{C}$ & 0.9800 \\
\hline $\mathrm{C} 8-\mathrm{C} 9$ & $1.406(4)$ & $\mathrm{C} 22-\mathrm{H} 22 \mathrm{~A}$ & 0.9800 \\
\hline $\mathrm{C} 8-\mathrm{C} 16$ & $1.444(4)$ & $\mathrm{C} 22-\mathrm{H} 22 \mathrm{~B}$ & 0.9800 \\
\hline $\mathrm{C} 9-\mathrm{O} 18$ & $1.350(3)$ & $\mathrm{C} 22-\mathrm{H} 22 \mathrm{C}$ & 0.9800 \\
\hline $\mathrm{C} 9-\mathrm{C} 10$ & $1.394(3)$ & $\mathrm{O} 23-\mathrm{C} 24$ & $1.430(4)$ \\
\hline $\mathrm{C} 10-\mathrm{C} 11$ & $1.383(4)$ & $\mathrm{O} 23-\mathrm{H} 23$ & $0.83(2)$ \\
\hline $\mathrm{C} 10-\mathrm{H} 10$ & 0.9500 & $\mathrm{C} 24-\mathrm{C} 25$ & $1.489(4)$ \\
\hline C11-H11 & 0.9500 & $\mathrm{C} 24-\mathrm{H} 24 \mathrm{~A}$ & 0.9900 \\
\hline $\mathrm{C} 12-\mathrm{H} 12 \mathrm{~A}$ & 0.9800 & $\mathrm{C} 24-\mathrm{H} 24 \mathrm{~B}$ & 0.9900 \\
\hline $\mathrm{C} 12-\mathrm{H} 12 \mathrm{~B}$ & 0.9800 & $\mathrm{C} 25-\mathrm{H} 25 \mathrm{~A}$ & 0.9800 \\
\hline $\mathrm{C} 12-\mathrm{H} 12 \mathrm{C}$ & 0.9800 & $\mathrm{C} 25-\mathrm{H} 25 \mathrm{~B}$ & 0.9800 \\
\hline $\mathrm{C} 13-\mathrm{O} 15$ & $1.205(3)$ & $\mathrm{C} 25-\mathrm{H} 25 \mathrm{C}$ & 0.9800 \\
\hline $\mathrm{C} 13-\mathrm{O} 14$ & $1.325(3)$ & & \\
\hline $\mathrm{C} 2-\mathrm{S} 1-\mathrm{C} 5$ & $89.55(12)$ & $\mathrm{N} 17-\mathrm{C} 16-\mathrm{C} 8$ & $178.3(3)$ \\
\hline $\mathrm{N} 3-\mathrm{C} 2-\mathrm{C} 6$ & $123.6(2)$ & $\mathrm{C} 9-\mathrm{O} 18-\mathrm{C} 19$ & $117.03(18)$ \\
\hline $\mathrm{N} 3-\mathrm{C} 2-\mathrm{S} 1$ & $114.17(18)$ & $\mathrm{O} 18-\mathrm{C} 19-\mathrm{C} 20$ & $108.51(19)$ \\
\hline $\mathrm{C} 6-\mathrm{C} 2-\mathrm{S} 1$ & $122.25(18)$ & $\mathrm{O} 18-\mathrm{C} 19-\mathrm{H} 19 \mathrm{~A}$ & 110.0 \\
\hline $\mathrm{C} 2-\mathrm{N} 3-\mathrm{C} 4$ & $111.7(2)$ & $\mathrm{C} 20-\mathrm{C} 19-\mathrm{H} 19 \mathrm{~A}$ & 110.0 \\
\hline $\mathrm{C} 5-\mathrm{C} 4-\mathrm{N} 3$ & $115.0(2)$ & $\mathrm{O} 18-\mathrm{C} 19-\mathrm{H} 19 \mathrm{~B}$ & 110.0 \\
\hline $\mathrm{C} 5-\mathrm{C} 4-\mathrm{C} 12$ & $126.3(2)$ & $\mathrm{C} 20-\mathrm{C} 19-\mathrm{H} 19 \mathrm{~B}$ & 110.0 \\
\hline $\mathrm{N} 3-\mathrm{C} 4-\mathrm{C} 12$ & $118.7(2)$ & $\mathrm{H} 19 \mathrm{~A}-\mathrm{C} 19-\mathrm{H} 19 \mathrm{~B}$ & 108.4 \\
\hline $\mathrm{C} 4-\mathrm{C} 5-\mathrm{C} 13$ & $128.6(2)$ & $\mathrm{C} 19-\mathrm{C} 20-\mathrm{C} 22$ & $111.8(2)$ \\
\hline $\mathrm{C} 4-\mathrm{C} 5-\mathrm{S} 1$ & $109.62(19)$ & $\mathrm{C} 19-\mathrm{C} 20-\mathrm{C} 21$ & $108.0(2)$ \\
\hline $\mathrm{C} 13-\mathrm{C} 5-\mathrm{S} 1$ & $121.80(19)$ & $\mathrm{C} 22-\mathrm{C} 20-\mathrm{C} 21$ & $110.5(2)$ \\
\hline $\mathrm{C} 7-\mathrm{C} 6-\mathrm{C} 11$ & $118.3(2)$ & $\mathrm{C} 19-\mathrm{C} 20-\mathrm{H} 20$ & 108.9 \\
\hline $\mathrm{C} 7-\mathrm{C} 6-\mathrm{C} 2$ & $121.3(2)$ & $\mathrm{C} 22-\mathrm{C} 20-\mathrm{H} 20$ & 108.9 \\
\hline $\mathrm{C} 11-\mathrm{C} 6-\mathrm{C} 2$ & $120.4(2)$ & $\mathrm{C} 21-\mathrm{C} 20-\mathrm{H} 20$ & 108.9 \\
\hline $\mathrm{C} 8-\mathrm{C} 7-\mathrm{C} 6$ & $120.7(2)$ & $\mathrm{C} 20-\mathrm{C} 21-\mathrm{H} 21 \mathrm{~A}$ & 109.5 \\
\hline $\mathrm{C} 8-\mathrm{C} 7-\mathrm{H} 7$ & 119.7 & $\mathrm{C} 20-\mathrm{C} 21-\mathrm{H} 21 \mathrm{~B}$ & 109.5 \\
\hline $\mathrm{C} 6-\mathrm{C} 7-\mathrm{H} 7$ & 119.7 & $\mathrm{H} 21 \mathrm{~A}-\mathrm{C} 21-\mathrm{H} 21 \mathrm{~B}$ & 109.5 \\
\hline $\mathrm{C} 7-\mathrm{C} 8-\mathrm{C} 9$ & $120.6(2)$ & $\mathrm{C} 20-\mathrm{C} 21-\mathrm{H} 21 \mathrm{C}$ & 109.5 \\
\hline $\mathrm{C} 7-\mathrm{C} 8-\mathrm{C} 16$ & $119.8(2)$ & $\mathrm{H} 21 \mathrm{~A}-\mathrm{C} 21-\mathrm{H} 21 \mathrm{C}$ & 109.5 \\
\hline $\mathrm{C} 9-\mathrm{C} 8-\mathrm{C} 16$ & $119.6(2)$ & $\mathrm{H} 21 \mathrm{~B}-\mathrm{C} 21-\mathrm{H} 21 \mathrm{C}$ & 109.5 \\
\hline $\mathrm{O} 18-\mathrm{C} 9-\mathrm{C} 10$ & $125.0(2)$ & $\mathrm{C} 20-\mathrm{C} 22-\mathrm{H} 22 \mathrm{~A}$ & 109.5 \\
\hline $\mathrm{O} 18-\mathrm{C} 9-\mathrm{C} 8$ & $116.4(2)$ & $\mathrm{C} 20-\mathrm{C} 22-\mathrm{H} 22 \mathrm{~B}$ & 109.5 \\
\hline $\mathrm{C} 10-\mathrm{C} 9-\mathrm{C} 8$ & $118.6(2)$ & $\mathrm{H} 22 \mathrm{~A}-\mathrm{C} 22-\mathrm{H} 22 \mathrm{~B}$ & 109.5 \\
\hline $\mathrm{C} 11-\mathrm{C} 10-\mathrm{C} 9$ & $120.1(2)$ & $\mathrm{C} 20-\mathrm{C} 22-\mathrm{H} 22 \mathrm{C}$ & 109.5 \\
\hline $\mathrm{C} 11-\mathrm{C} 10-\mathrm{H} 10$ & 119.9 & $\mathrm{H} 22 \mathrm{~A}-\mathrm{C} 22-\mathrm{H} 22 \mathrm{C}$ & 109.5 \\
\hline $\mathrm{C} 9-\mathrm{C} 10-\mathrm{H} 10$ & 119.9 & $\mathrm{H} 22 \mathrm{~B}-\mathrm{C} 22-\mathrm{H} 22 \mathrm{C}$ & 109.5 \\
\hline $\mathrm{C} 10-\mathrm{C} 11-\mathrm{C} 6$ & $121.6(2)$ & $\mathrm{C} 24-\mathrm{O} 23-\mathrm{H} 23$ & $111(3)$ \\
\hline
\end{tabular}




\begin{tabular}{llll}
$\mathrm{C} 10-\mathrm{C} 11-\mathrm{H} 11$ & 119.2 & $\mathrm{O} 23-\mathrm{C} 24-\mathrm{C} 25$ & $109.5(2)$ \\
$\mathrm{C} 6-\mathrm{C} 11-\mathrm{H} 11$ & 119.2 & $\mathrm{O} 23-\mathrm{C} 24-\mathrm{H} 24 \mathrm{~A}$ & 109.8 \\
$\mathrm{C} 4-\mathrm{C} 12-\mathrm{H} 12 \mathrm{~A}$ & 109.5 & $\mathrm{C} 25-\mathrm{C} 24-\mathrm{H} 24 \mathrm{~A}$ & 109.8 \\
$\mathrm{C} 4-\mathrm{C} 12-\mathrm{H} 12 \mathrm{~B}$ & 109.5 & $\mathrm{O} 23-\mathrm{C} 24-\mathrm{H} 24 \mathrm{~B}$ & 109.8 \\
$\mathrm{H} 12 \mathrm{~A}-\mathrm{C} 12-\mathrm{H} 12 \mathrm{~B}$ & 109.5 & $\mathrm{C} 25-\mathrm{C} 24-\mathrm{H} 24 \mathrm{~B}$ & 109.8 \\
$\mathrm{C} 4-\mathrm{C} 12-\mathrm{H} 12 \mathrm{C}$ & 109.5 & $\mathrm{H} 24 \mathrm{~A}-\mathrm{C} 24-\mathrm{H} 24 \mathrm{~B}$ & 108.2 \\
$\mathrm{H} 12 \mathrm{~A}-\mathrm{C} 12-\mathrm{H} 12 \mathrm{C}$ & 109.5 & $\mathrm{C} 24-\mathrm{C} 25-\mathrm{H} 25 \mathrm{~A}$ & 109.5 \\
$\mathrm{H} 12 \mathrm{~B}-\mathrm{C} 12-\mathrm{H} 12 \mathrm{C}$ & 109.5 & $\mathrm{C} 24-\mathrm{C} 25-\mathrm{H} 25 \mathrm{~B}$ & 109.5 \\
$\mathrm{O} 15-\mathrm{C} 13-\mathrm{O} 14$ & $123.9(2)$ & $\mathrm{H} 25 \mathrm{~A}-\mathrm{C} 25-\mathrm{H} 25 \mathrm{~B}$ & 109.5 \\
$\mathrm{O} 15-\mathrm{C} 13-\mathrm{C} 5$ & $123.5(2)$ & $\mathrm{C} 24-\mathrm{C} 25-\mathrm{H} 25 \mathrm{C}$ & 109.5 \\
$\mathrm{O} 14-\mathrm{C} 13-\mathrm{C} 5$ & $112.7(2)$ & $\mathrm{H} 25 \mathrm{~A}-\mathrm{C} 25-\mathrm{H} 25 \mathrm{C}$ & 109.5 \\
$\mathrm{C} 13-\mathrm{O} 14-\mathrm{H} 14$ & $113(3)$ & $\mathrm{H} 25 \mathrm{~B}-\mathrm{C} 25-\mathrm{H} 25 \mathrm{C}$ & 109.5 \\
\hline
\end{tabular}

Hydrogen-bond geometry $\left(\AA,{ }^{\circ}\right)$

\begin{tabular}{lllll}
\hline$D-\mathrm{H} \cdots A$ & $D-\mathrm{H}$ & $\mathrm{H} \cdots A$ & $D \cdots A$ & $D-\mathrm{H} \cdots A$ \\
\hline $\mathrm{O} 23-\mathrm{H} 23 \cdots \mathrm{N} 3^{\mathrm{i}}$ & $0.83(2)$ & $2.07(2)$ & $2.878(3)$ & $162(4)$ \\
$\mathrm{O} 14-\mathrm{H} 14 \cdots \mathrm{O} 23^{\mathrm{ii}}$ & $0.84(2)$ & $1.80(2)$ & $2.631(3)$ & $170(4)$ \\
\hline
\end{tabular}

Symmetry codes: (i) $-x+1, y+1 / 2,-z+1$; (ii) $x-1, y, z$. 